\title{
AUTOSAR Appropriates Functional Safety and Multi-core Exploitation
}

\author{
Bert Böddeker ${ }^{1}$ and Rafael Zalman ${ }^{2}$ \\ 1 DENSO AUTOMOTIVE Deutschland GmbH \\ Eching, Germany \\ 2 Infineon Technologies AG \\ Automotive Electronics \\ Munich, Germany
}

\begin{abstract}
The main subject of this presentation is the connection between AUTOSAR as software standardization initiative and the automotive functional safety domain. The History of AUTOSAR and the functional safety evolution for road vehicles will be presented together with a short overview of the typical implementation of these techniques (E-Gas, X by Wire, etc.).

New emerging multi-core architectures are influencing the automotive related software implementations and have relevance in both software standardization and in the implementation of specific functional safety techniques. The actual status will be presented together with the potential future techniques.

It is discussed how much support AUTOSAR can already provide for practical implementations of functional safety and multi core today followed by an outlook on ongoing research and further standardization needs.
\end{abstract}

